\title{
Novel identification of zyxin upregulations in the motile phenotype of hepatocellular carcinoma
}

\author{
Shirley M-H Syㅜㄹ Paul B-S Lai ${ }^{2}$, Etonia Pang ${ }^{1}$, Navy L-Y Wong ${ }^{1}$, Ka-Fai To ${ }^{1}$, \\ Philip J Johnson ${ }^{3}$ and Nathalie Wong ${ }^{1}$ \\ ${ }^{1}$ Department of Anatomical and Cellular Pathology, Chinese University of Hong Kong, Shatin, N.T., SAR \\ Hong Kong, China: ${ }^{2}$ Department of Surgery, Chinese University of Hong Kong, Shatin, N.T., SAR Hong Kong, \\ China and ${ }^{3}$ Institute for Cancer Studies, University of Birmingham, Birmingham, UK
}

\begin{abstract}
Genome-wide copy number aberrations are common in hepatocellular carcinoma, although the precise genetic events underlying disease progression remain poorly defined. Previous work from our group has indicated several regional chromosomal gains such as chromosome 7q34-q36 that are associated with advanced metastatic tumors. Although the distal chromosome $7 q$ gains have also been implicated in the progression of other malignancies, information on underlying targeted genes is limited. In this study, we have examined the chromosome 7q34-q36 region for involved gene(s) (or genes of interest). An integrated array-based comparative genomic hybridization and transcriptional mapping analyses has enabled us to identify a single candidate, zyxin on chromosome 7q34-q36. This array-derived finding was supported by quantitative reverse transcription-polymerase chain reaction, which also indicated common upregulations of zyxin in hepatocellular carcinoma tumors compared to their corresponding nonmalignant liver tissue (17/52 cases; 33\%). Although there was no correlation between zyxin expression and tumor stagings, there was a significant increase in messenger RNA levels in hepatocellular carcinoma cases that presented with multifocal disease $(211.5 \pm 936.9$ fold) compared to those with solitary lesions (3.5 \pm 6.3 -fold). Moreover, recurrence after resection was common in cases that displayed zyxin overexpressions in the initial resected tumor $(P=0.05)$. Functional examination of zyxin by small interfering RNA-mediated knockdown in Hep3B cell line indicated a significant inhibition on cell migration through porous membrane $(P=0.002)$ and invasion through matrigel-coated membrane $(P=0.005)$. In summary, mapping of chromosome 7q34-q36 has led to the identification of frequent zyxin overexpressions in hepatocellular carcinoma, and a potential role for zyxin in conferring a motile phenotype.
\end{abstract}

Modern Pathology (2006) 19, 1108-1116. doi:10.1038/modpathol.3800626; published online 5 May 2006

Keywords: hepatocellular carcinoma; complementary DNA-based comparative genomic hynridization; transcriptional mapping; zyxin; cell migration; cell invasion

Extensive genetic characterizations on hepatocellular carcinoma have indicated common copy number gains on chromosomes $1 \mathrm{q}, 8 \mathrm{q}, 17 \mathrm{q}$ and $20 \mathrm{q}$, and losses on $1 p, 4 q$ 8p, 13q, 16q and $17 p .{ }^{1-3}$ While these changes have been considered early genetic events in liver carcinogenesis, the molecular events by which specific genomic alterations contribute to the progression of hepatocellular carcinoma remain elusive. Our group has previously conducted largescale correlative analysis on the genomic data

Correspondence: Dr N Wong, D Phil, Department of Anatomical and Cellular Pathology, Chinese University of Hong Kong, Prince of Wales Hospital, Shatin, N.T., SAR Hong Kong, China.

E-mail: natwong@cuhk.edu.hk

Received 24 January 2006; revised 10 April 2006; accepted 11 April 2006; published online 5 May 2006 derived from early and advanced stages hepatocellular carcinoma tumors with an aim to define the genetic aberrations involved in disease progression. ${ }^{4}$ A number of regional copy gains were indicated, among which chromosome 7q34-q36 gains were common in the advanced metastatic hepatocellular carcinoma. ${ }^{4}$

Distal chromosome 7q gains have been suggested in association with biliary tract carcinoma progression, ${ }^{5}$ and higher-stage non-small cell lung cancer with nodal involvement. ${ }^{6}$ Previous studies have also demonstrated a positive association between prostate cancer progression and copy gains of distal chromosome $7 \mathrm{q},{ }^{7}$ and the amplification of chromosome $7 q 31-q 36$ region in the progression of gliomas to grade III or IV. ${ }^{8}$ Taken together, it is likely that gene(s) residing on chromosome 7q34-q36 may have 
a role in cancer progression. In the current study, a combined analysis using of high-resolution genomic and transcriptional mapping was performed to identify potential tumor-related gene(s) residing on chromosome 7q34-q36. Using a complementary DNA array platform with an average resolution of $\sim 91 \mathrm{~kb}$ on chromosome 7 , mapping studies where performed on a hepatocellular carcinoma tumor that displayed regional chromosome 7q34-q36 highlevel gains from metaphase comparative genomic hybridization analysis. This integrated analysis has permitted recognition of candidate genes and minimized the number of genes required for subsequent quantitative reverse transcription-polymerase chain reaction validations. The functional effects of an identified candidate gene on hepatocellular carcinoma cell proliferation and motility were further investigated.

\section{Materials and methods}

\section{Patients}

Tumorous hepatocellular carcinoma and the corresponding adjacent nonmalignant liver tissues were obtained from 52 patients who underwent curative surgery for hepatocellular carcinoma at the Prince of Wales Hospital, Hong Kong between 1997 and 2002. Histological examination confirmed the diagnosis of hepatocellular carcinoma, and the presence of underlying cirrhosis in $\mathbf{8 0 . 8 \%}$. Serological analysis indicated chronic viral hepatitis B carriage in 94.0\% of patients. The American Joint Cancer Center tumor staging system classified one case as T1, 30 cases as T2, 12 cases as T3 and 9 cases as T4. Statistical analysis did not suggest differences in the clinicopathological characteristics between the early T1/T2 and advanced T3/T4 stage patients $(P>0.05)$, except for the presence of multifocal feature $(P<0.0001)$ (Table 2). Five year follow-up information on 32 cases showed tumor recurrence in 15 patients.

\section{Complementary DNA Microarray-Based Comparative Genomic Hybridization}

The complementary DNA microarray chip utilized contained 1738 complementary DNA clones that spanned an average resolution of $\sim 91 \mathrm{~kb}$ along the entire human chromosome 7 (Ontario Cancer Institute, Toronto, Canada). Complementary DNA microarray-based comparative genomic hybridization was performed according to method described in Beheshti et al. ${ }^{9}$ A hepatocellular carcinoma cell line, HKCI-6, that displayed high-level gains of regional chromosome 7q34-q36 was employed in the mapping analyses. HKCI-6 was established from the tumorous liver tissue of a chronic hepatitis B virus infected male patient, who presented a stage T3 hepatocellular carcinoma tumor with multifocal lesions. DNA from HKCI-6, and a reference pool (lymphocytic DNA from three healthy subjects) were differentially labeled with Cy5-dCTP and Cy3-dCTP using the RadPrime DNA labeling system (Gibco Invitrogen Corporation). Labeled DNA was combined with $30 \mu \mathrm{g}$ human Cot-1 DNA, $20 \mu \mathrm{g}$ poly (dA-dT) and $100 \mu \mathrm{g}$ yeast tRNA in DIGhyb buffer prior to overnight hybridization in a dark chamber at $37^{\circ} \mathrm{C}$. Following posthybridization washes in $1 \times$ saline sodium citrate $/ 0.1 \%$ sodium dodecyl sulfate at $45^{\circ} \mathrm{C}$ and gentle rinsing in $1 \times$ saline sodium citrate at room temperature, hybridized signals were captured by the ScanArray 5000 scanner (GSI Lumonics, Billerica, MA) and analyzed by the GenePix Pro 3.0. Data manipulation was facilitated using the Normalization Suite v1.63 (http://www. utoronto.ca/cancyto/). Results from duplicate spots and dye swap experiments were calculated and incorporated with the physical map locations of complementary DNA in sequential order of megabase distances. 'Test to reference' ratios greater than twofold were considered as copy number gain.

\section{Expression Microarray}

Using complementary DNA microarray chip as the array-comparative genomic hybridization study (Ontario Cancer Institute, Toronto, Canada), expression array analysis was performed according to procedure previously described from our group. ${ }^{10}$ Ten micrograms of total RNA from HKCI-6 and reference pool (3 normal livers acquired from CloneTech, Stratagene and Ambion) were reverse transcribed by AncT messenger RNA primer using Supescript II reverse transcriptase separately (Gibco Invitrogen Corporation). Complementary DNA differentially labeled with Cy3-dCTP and Cy5-dCTP were combined with $20 \mu \mathrm{g}$ calf thymus DNA and $100 \mu \mathrm{g}$ yeast tRNA prior to hybridization onto array slides. Following posthybridization washes, image acquisition and data analysis were as described for complementary DNA microarray-based comparative genomic hybridization. 'Test to reference' ratios greater than twofold were considered as upregulations.

\section{Quantitative Reverse Transcription-Polymerase Chain Reaction}

Total RNA extracted from HKCI-6 and primary hepatocellular carcinoma tumors using TRIzol reagent (Gibco Invitrogen Corporation) were treated with 1.0 U Rq1 RNase-free DNase (Promega Corporation) prior to reverse transcription. To ensure no genomic DNA carryover, no-reverse transcription polymerase chain reaction was first performed using primer sets for genomic beta-globin gene (sense: $5^{\prime}$ GAA GAG CCA AGG ACA GGT AC- $3^{\prime}$ and antisense: $5^{\prime}$-CAA CTT CAT CCA CGT TCA CC- $3^{\prime}$ ). First strand complementary DNA was prepared from total RNA using the random hexanucleotide primer and Taq- 
Man Reverse Transcription Reagent (Applied Biosystems). Primers used for zyxin were (sense) $5^{\prime}$ CAC CGG CTC AGA ACC AAA-3' (antisense) 5'-TGC TCC ATG TCC TGC ATT AG-3' ${ }^{\prime}$ and for ATP-binding cassette sub-family $F$ member 2 were (sense) 5'-ACA ACG TGT GCA CCC TGA-3' (antisense) 5'-CCA GCT GCT CAA ACA CGT C-3', respectively. Primers for the internal control glyceraldehyde-3-phosphate dehydrogenase were (sense) 5'-ATG GGT GTG AAC CAT GAG AAG-3' and (antisense) $5^{\prime}$-AGT TGT CAT GGA TGA CCT TGG-3'. The emission intensity from SYBR green binding to doublestranded DNA was detected by the iCycler detection system (BioRad Laboratories, Hercules, CA). Triplicate PCR amplifications were performed for each sample. No template polymerase chain reactions served as negative controls in all experiments. Relative expression levels in primary tumors with reference to normal liver RNA from three individuals were expressed as $2^{-\Delta \Delta C_{\mathrm{t}}}$. Expression levels greater than twofold were considered as up-regulation.

\section{Expression Knockdown by RNA Interference}

Hep3B was cultured in Dulbecco's modified eagle medium according to recommendation from the American Tissue and Cell Collection (USA). Specific post-transcriptional knockdown of zyxin expression were achieved using the SMART small interfering RNA pool (Dharmacon Research Inc., USA). A siControl pool that does not align to any known mammalian mRNA served as nonspecific small interfering RNA in mock experiments. Control experiment using transfectant only was also performed. Cells at exponential growth were transiently transfected using Lipofectamine 2000 in fetal bovine serum-free medium. After $5 \mathrm{~h}$ incubation, transfection mixtures were replaced with growth medium.

\section{Cell Proliferation Assay}

Cell viability was assessed using the thiazolyl blue tetrazolium bromide assay. Approximately $6 \times 10^{3}$ cells were seeded in quintuplicate onto 96-well plates at day 0 . The assay was performed at $24 \mathrm{~h}$ intervals. Following removal of medium, $50 \mu \mathrm{l}$ of $1.0 \mathrm{mg} / \mathrm{ml}$ thiazolyl blue tetrazolium bromide was added to the cells and incubated at $37^{\circ} \mathrm{C}$ for $4 \mathrm{~h}$. Using SPECTRA Rainbow v2.02 plate reader (TECAN Austria Ges.m.b.H), cell viability was measured by the colorimetric product developed at absorbance $570 \mathrm{~nm}$. Results were averaged from three independent experiments.

\section{TdT-Mediated dUTP Nick End Labeling Assay}

Transfected cells were fixed in $4 \%$ paraformaldehyde and permeabilized with $0.2 \%$. Triton X-100 prior to fluorescein-12-dUTP incorporation using the TdT-mediated dUTP nick end labeling assay (Promega Corporation). The reaction took place in a dark chamber at $37^{\circ} \mathrm{C}$ for $1 \mathrm{~h}$. Slides washed in $2 \times$ saline sodium citrate were nuclear stained with 4',6-diamidino-2-phenylindole. The evaluation of fluorescent signals for apoptotic cells was performed on a Leitz DM RB fluorescence microscope (Leica Microsystems Ltd.).

\section{Cell Migration and Invasion Assays}

The cell migration capacity was estimated by the Costar transwell inserts (8 $\mu \mathrm{m}$ pores) (Corning Incorporation) and the cell invasive capacity was estimated using the BD BioCoat Matrigel invasion chambers ( $8 \mu \mathrm{m}$ pores, 24-wells) (BD Bioscience). Cells $\left(1 \times 10^{4}\right.$ for migration study and $5 \times 10^{4}$ for invasion assay) were resuspended in serum-free medium and seeded onto the top chamber of prewet inserts. After $48 \mathrm{~h}$ incubation, cells on top of the membrane were wiped off and the inserts were methanol fixed, stained with hematoxylin and eosin, and alcohol dehydrated. Numbers of cells invaded to the underside of the membrane were scored from five microscope fields (magnification, $\times 100$ ). Each condition was performed in triplicates and results described were the average from two independent experiments.

\section{Statistical Analysis}

Correlation of gender, cirrhosis, hepatitis B viral status and multifocal presentation between early and advanced stage hepatocellular carcinoma were examined using Pearson's $\chi^{2}$ test. The Student's $t$-test was performed to compare the differences in age and serum alpha-fetoprotein level. The expression levels of candidate gene(s) between different groups of hepatocellular carcinoma tumors were compared by Student's $t$-test and $\chi^{2}$ test. Results derived from cell proliferation assays were examined using one-way analysis of variance. In invasion and migration assays, Student's $t$-test was applied to investigate potential effects upon treatments. A difference was considered significant when $P$-value $\leq 0.05$. All statistical analysis was performed using SPSS for Windows 10.0 software and Graphpad Prism 3.0.

\section{Results}

\section{Candidate Genes on Chromosome 7q34-q36}

Simultaneous genomic and transcriptional mapping analyses on chromosome 7 were performed on HKCI-6 using the complementary DNA microarray platform. Comparing the profiles derived from metaphase comparative genomic hybridization with the complementary DNA microarray-based comparative genomic hybridization, a concordant copy gain in 
the region chromosome $7 q 34-q 36$ was indicated (Figure 1a). A total of 27 expressed sequence tags along chromosome 7q34-q36 demonstrated copy number gains (microarray ratio $\geq 2.0$ ) (Table 1a), although parallel expression microarray analysis indicated the upregulations (microarray ratio $\geq 2.0$ ) of only 12 transcripts from within the same region (Table 1b). a

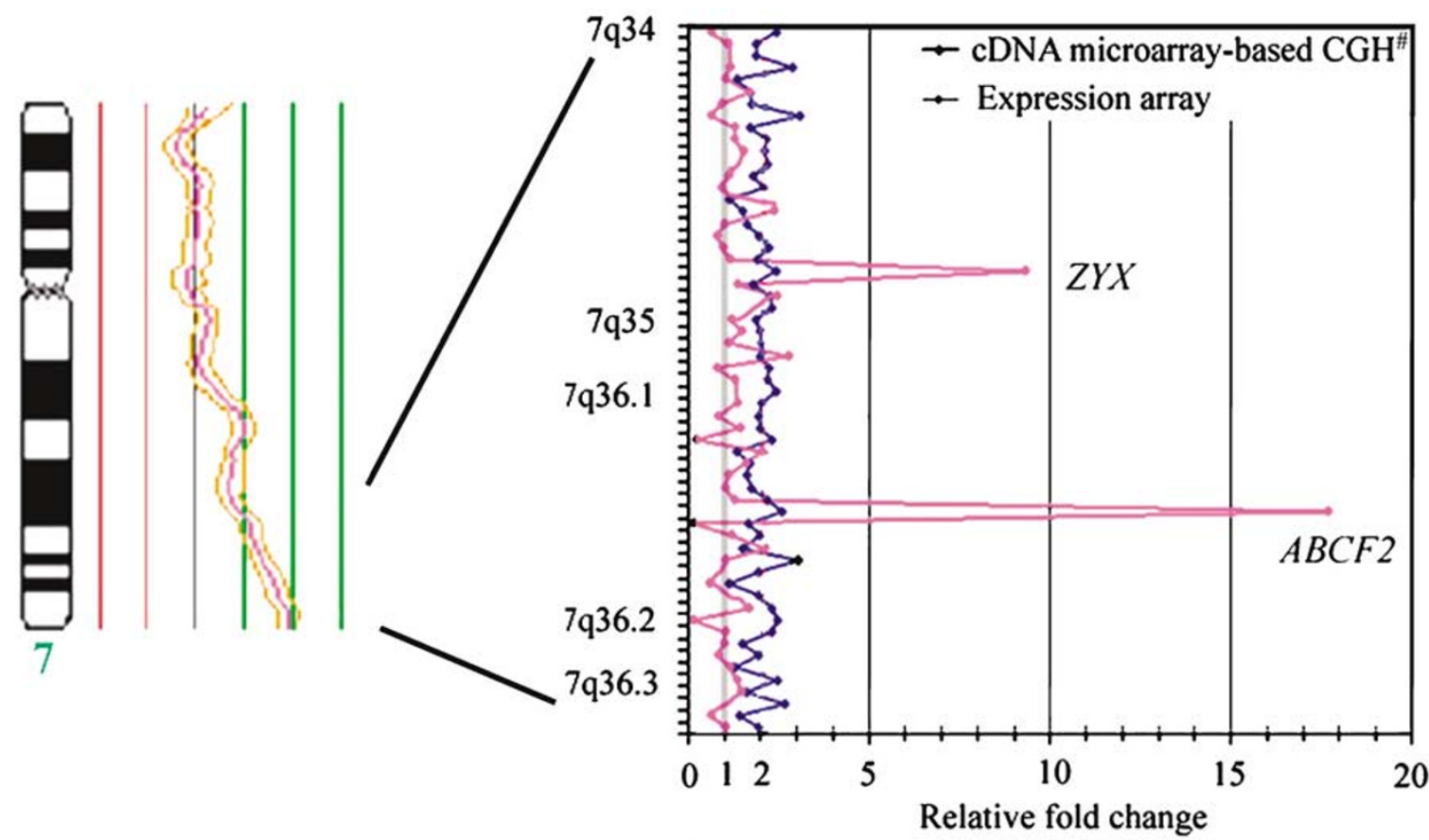

b

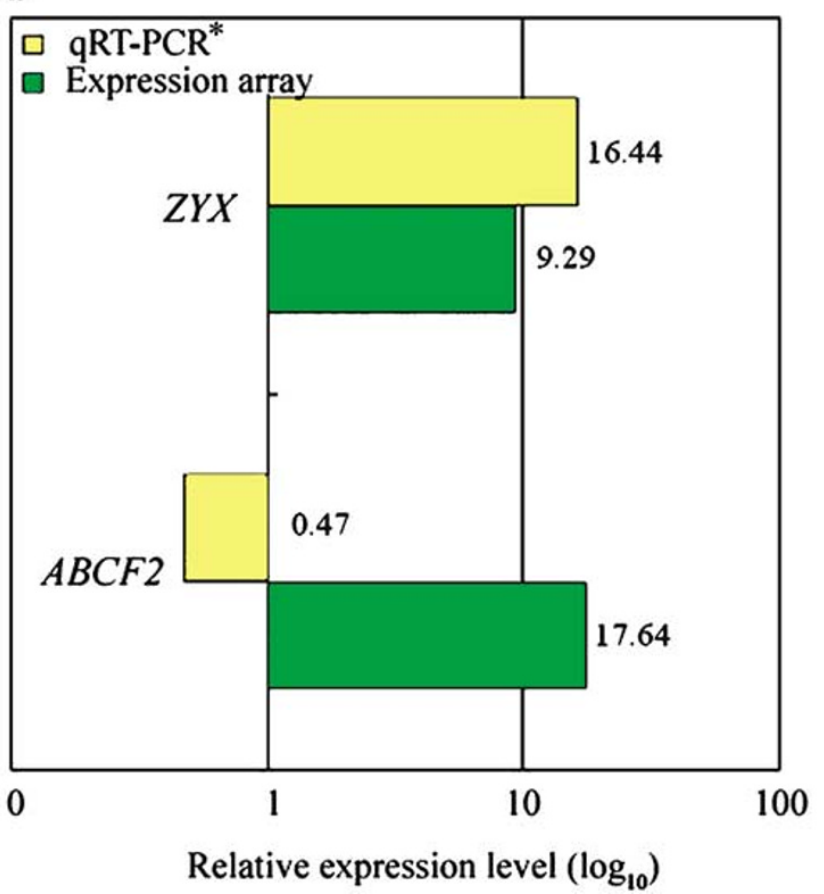

C

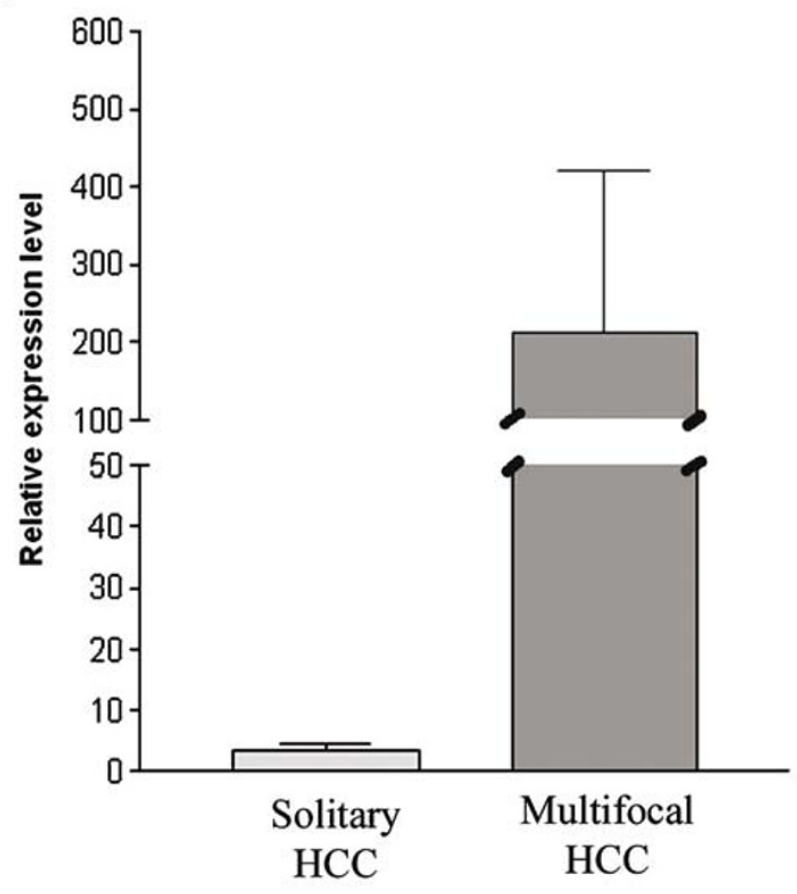

Figure 1 Integrative complementary DNA microarray-based comparative genomic hybridization and transcriptional mapping on chromosome 7q34-q36. (a) Left: Metaphase comparative genomic hybridization revealed a regional high-level gain of 7q34-q36 in HKCI6. Right: Combined complementary DNA microarray-based comparative genomic hybridization" and expression analyses identified two candidate genes, $Z Y X$ and $A B C F 2$, with increased copy gain and distinct over-expression. (b) Over-expression of messenger RNA level of $Z Y X$ was confirmed by quantitative reverse transcription-polymerase chain reaction* in HKCI-6. (c) Relative expression level of $Z Y X$ in hepatocellular carcinoma patients. ZYX expression was found to be higher in cases with multifocal tumor lesions than cases with solitary tumor. 
Table 1 Copy number gain and upregulated genes on chromosome 7q34-q36. (a) Genes that displayed copy number gains as depicted from array-based comparative genomic hybridization analysis. (b) Upregulated genes identified from expression array analysis. Genes selected for quantitative reverse transcription-polymerase chain reaction validation are in bold

\begin{tabular}{llll}
\hline Gene name & Gene symbol Accession no. Cytoband Microarray ratio
\end{tabular}

(a) Genes with copy number gain

cAMP responsive element binding protein 3-like 2

Homeodomain interacting protein kinase 2

KIAA1718 protein

Makorin, ring finger protein, 1

KIAA1277

NADH dehydrogenase (ubiquinone) 1 beta subcomplex, 2

Hypothetical protein FLJ40852

Single-stranded DNA binding protein 1

KIAA0773 gene product

\section{Zyxin}

EPH receptor A1

Hypothetical protein LOC154761

Protein disulfide isomerase family A, member 4

Hypothetical protein LOC155060

Likely ortholog of mouse SCO-spondin

Hypothetical gene LOC401431

Hypothetical protein MGC3036

LR8 protein

Hepatocellular carcinoma-associated antigen 112

Cyclin-dependent kinase 5

Solute carrier family 4 , anion exchanger, member 2

ATP-binding cassette, sub-family $F$, member 2

Protein kinase, AMP-activated, gamma 2 non-catalytic subunit

Myeloid/lymphoid or mixed-lineage leukemia 3

Dipeptidylpeptidase 6

Ring finger protein 32

Protein tyrosine phosphatase, receptor type, $\mathrm{N}$ polypeptide 2

(b) Upregulated genes

KIAA1277

T-cell receptor beta constant 1

KIAA0773 gene product

\section{Zyxin}

EPH receptor A1

Protein disulfide isomerase family A, member 4

Amiloride binding protein 1

Cyclin-dependent kinase 5

ATP-binding cassette, sub-family F, member 2

Ras homolog enriched in brain

Myeloid/lymphoid or mixed-lineage leukemia 3

Ring finger protein 32

Accession no.

\begin{tabular}{|c|c|c|c|}
\hline CREB3L2 & AA033986 & $7 q 34$ & 2.42 \\
\hline HIPK2 & R12041 & $7 q 34$ & 2.10 \\
\hline KIAA1718 & R16961 & $7 q 34$ & 3.07 \\
\hline MKRN1 & BG484337 & $7 q 34$ & 2.13 \\
\hline KIAA1277 & AA210973 & $7 q 34$ & 2.86 \\
\hline NDUFB2 & R07689 & $7 q 34$ & 2.78 \\
\hline FLJ40852 & Т95526 & $7 q 34$ & 8.27 \\
\hline SSBP1 & W68198 & $7 q 34$ & 2.05 \\
\hline KIAA0773 & BG819654 & $7 q 35$ & 2.59 \\
\hline$Z Y X$ & R76163 & $7 q 35$ & 2.41 \\
\hline EPHA1 & W39186 & $7 q 35$ & 2.25 \\
\hline LOC154761 & BG678683 & $7 q 35$ & 2.29 \\
\hline ERP70 & BG818939 & $7 q 36.1$ & 2.01 \\
\hline LOC155060 & Н08319 & $7 q 36.1$ & 2.24 \\
\hline KIAA0543 & Н39161 & $7 q 36.1$ & 2.17 \\
\hline LOC401431 & R12636 & $7 q 36.1$ & 2.44 \\
\hline MGC3036 & R36051 & $7 q 36.1$ & 2.03 \\
\hline LR8 & H15021 & 7q36.1 & 2.32 \\
\hline HCA112 & H60213 & 7q36.1 & 3.93 \\
\hline CDK5 & H87204 & 7q36.1 & 2.73 \\
\hline SLC4A2 & R71723 & $7 q 36.1$ & 2.18 \\
\hline$A B C F 2$ & W25202 & $7 q 36.1$ & 2.58 \\
\hline PRKAG2 & H64260 & $7 q 36.1$ & 3.03 \\
\hline MLL3 & H73223 & $7 q 36.1$ & 4.34 \\
\hline DPP6 & R88469 & $7 q 36.2$ & 2.30 \\
\hline RNF32 & R60654 & $7 q 36.3$ & 2.54 \\
\hline PTPRN2 & H06958 & $7 q 36.3$ & 2.65 \\
\hline KIAA1277 & AA210973 & $7 q 34$ & 2.49 \\
\hline TRBC1 & AA147693 & $7 q 34$ & 2.36 \\
\hline KIAA0773 & H20381 & $7 q 35$ & 3.01 \\
\hline$Z Y X$ & $R 76163$ & $7 q 35$ & 9.29 \\
\hline EPHA1 & W39186 & 7q35 & 2.41 \\
\hline ERP70 & AA035349 & 7q36.1 & 3.79 \\
\hline ABP1 & Т53433 & 7q36.1 & 2.03 \\
\hline CDK5 & H87204 & 7q36.1 & 2.96 \\
\hline$A B C F 2$ & W25202 & $7 q 36.1$ & 17.64 \\
\hline RHEB & W01907 & $7 q 36.1$ & 2.11 \\
\hline MLL3 & N78369 & $7 q 36.1$ & 2.28 \\
\hline RNF32 & R60654 & $7 q 36.3$ & 4.12 \\
\hline
\end{tabular}

When aligning the results from genomic mapping and transcriptional analysis, nine transcripts demonstrated simultaneous DNA copy number gains and messenger RNA upregulations. These included KIAA1277, KIAA0773, zyxin (ZYX), EPH receptor A1 (EPHA1), protein disulfide isomerase family $A$ member 4 (ERP70), cyclin-dependent kinase 5 (CDK5), ATP-binding cassette sub-family $F$ member 2 (ABCF2), mixed-lineage leukemia 3 (MLL3) and ring finger protein 32 (RNF32). In particular, $Z Y X$ and $A B C F 2$ displayed distinct over-expressions at 9.3-fold and 17.6-fold, respectively, compared to other candidates (Figure 1a). Further quantitative reverse transcription-polymerase chain reaction verification in the same specimen, however, confirmed the overexpression of only $Z Y X$ at 16.4 -fold, whereas contrary to array finding a downregulation of $A B C F 2$ was indicated (Figure 1b).

\section{Expression of ZYX mRNA in Primary Hepatocellular Carcinoma Tumors}

The $Z Y X$ expression was further examined in a cohort of 52 hepatocellular carcinoma tumors and the corresponding adjacent nonmalignant liver tissue (31 early stages T1/T2 and 21 advanced stages T3/T4) (Table 2). Overexpression of $Z Y X$ was 
Table 2 Clinicopathological information of 52 hepatocellular carcinoma patients

\begin{tabular}{|c|c|c|c|}
\hline & $\begin{array}{l}\text { Early T1/T2 } \\
\text { hepatocellular } \\
\text { carcinoma } \\
(\mathrm{n}=31)\end{array}$ & $\begin{array}{l}\text { Advanced T3/T4 } \\
\text { hepatocellular } \\
\text { carcinoma } \\
(\mathrm{n}=21)\end{array}$ & P-value \\
\hline $\begin{array}{l}\text { Age } \\
\text { Median } \\
\text { (range) }\end{array}$ & $56.0(40-84)$ & $56.0(24-75)$ & 0.481 \\
\hline $\begin{array}{l}\text { Gender } \\
\text { Male } \\
\text { Female }\end{array}$ & $\begin{array}{r}26(83.9 \%) \\
5(16.1 \%)\end{array}$ & $\begin{array}{r}17(81.0 \%) \\
4(19.0 \%)\end{array}$ & 1.000 \\
\hline $\begin{array}{l}\text { Alpha-fetoprotei } \\
\text { Median } \\
\text { (range) (ng/ml) }\end{array}$ & $n^{\mathrm{a}} 48.0(0.0-5989.0)$ & $331.0(4.0-11200.0)$ & 0.097 \\
\hline $\begin{array}{l}\text { Cirrhosis } \\
\text { Yes } \\
\text { No }\end{array}$ & $\begin{array}{r}26(83.9 \%) \\
5(16.1 \%)\end{array}$ & $\begin{array}{c}16(76.20 \%) \\
5(23.8 \%)\end{array}$ & 0.490 \\
\hline $\begin{array}{l}\text { HbsAg status }{ }^{\mathrm{a}} \\
\text { Yes } \\
\text { No }\end{array}$ & $\begin{array}{c}28(93.3 \%) \\
2(6.7 \%)\end{array}$ & $\begin{array}{c}19(95.0 \%) \\
1(5.0 \%)\end{array}$ & 0.490 \\
\hline $\begin{array}{l}\text { Multifocal preser } \\
\text { Yes } \\
\text { No }\end{array}$ & $\begin{array}{l}\text { atation } \\
\quad 3(9.7 \%) \\
28(90.3 \%)\end{array}$ & $\begin{array}{r}17(81.0 \%) \\
4(19.0 \%)\end{array}$ & $<0.0005$ \\
\hline
\end{tabular}

${ }^{\mathrm{a}}$ Applicable to cases with available information.

suggested in $\sim 33 \%$ cases $(17 / 52$ cases) with an average fold gain of $83.5 \pm 581.0$ compared to their nontumorous counterpart. Although correlative analysis did not suggest differential $Z Y X$ expressions with tumor staging $(P>0.05), Z Y X$ expression was found to be on average 60-fold higher in cases that presented with multiple tumor lesions $(n=20)$ $(211.5 \pm 936.9$-fold $)$ when compared with those presenting with solitary tumors $(n=32,3.5 \pm 6.3$ fold, Figure 1c). Furthermore, among the 32 patients in whom 5 year follow-up was available, a substantial portion of those with recurrent disease (14/ 15 cases) had overexpression of $Z Y X$ by $>10$-fold compared to those nonrecurring patients $(P=0.05)$.

\section{Effect of ZYX Knockdown on Hepatocellular Carcinoma Cells}

The Hep3B cell line was employed in the functional study of $Z Y X$ since Hep3B displayed a motile invasive phenotype and at the same time demonstrated an over-expression of $Z Y X$ (21.7-fold from quantitative reverse transcription-polymerase chain reaction analysis). More than $80 \%$ reduction in the messenger RNA expression of $Z Y X$ was achieved in Hep3B cells by RNA interference (siZYX). Quantitative reverse transcription-polymerase chain reaction also indicated the inhibition of $Z Y X$ expression to be maintained for up to 7 days. There was no observable change in cell morphology upon siZYX transfection compared to mock and control experiments, although a reduction in the relative cell proliferation by $\sim 30 \%$ in siZYX-transfected cells was indicated compared to mock experiments (Figure 2a). Analysis of knockdown effect on apoptosis by TdT-mediated dUTP nick end labeling NEL assay, on the other hand, did not reveal differences between siZYX, mock and control experiments (Figure 2b).

There was a marked difference in the invasion index of siZYX-treated cells and mock at 43.8 and 9.5 relative to control $(P=0.005)$ (Figure 3a). Similarly, in the migration assay, a significant reduction of chemotatic cell migration of siZYX-treated cells was found ( $P=0.002)$ (Figure $3 \mathrm{~b}$ and $\mathrm{c}$ ). The migration index of siZYX-transfected cells, and mock were 62.1 and 33.4 , respectively compared to control experiment.

\section{Discussion}

Metastasis remains the major obstacle to curative therapies for patients with hepatocellular carcinoma. Moreover, the high incidence of postoperative recurrence associated suggests the presence of preexisting micro-metastasis prior to surgery. ${ }^{11}$ This observation underscores the need to understand the molecular characteristics and related biological mechanisms that lead to tumor cell dissemination. In this study, we have attempted to map the chromosome 7q34-q36 region for potential candidate(s) that may be involved in hepatocellular carcinoma progression. Integrative positional mapping by array-based comparative genomic hybridization and expression array has enabled us to identify a single candidate gene, $Z Y X$. In order to confirm upregulation of $Z Y X$, its expression was examined in a cohort of hepatocellular carcinoma tumors among which $Z Y X$ over-expressions occurred in around $33 \%$ of cases when compared to the adjacent cirrhotic or fibrotic liver tissues. Overexpression of $Z Y X$ has also been demonstrated in melanoma, compared to its biological precursor melanocytes. ${ }^{12}$ However, although a relationship between $Z Y X$ upregulation and progression of bladder cancer was suggested, ${ }^{13}$ our correlative analysis did not demonstrate significant association between messenger RNA levels of $Z Y X$ and advanced stage hepatocellular carcinoma tumors. Rather, expression levels of $Z Y X$ in patients with multiple tumor lesions were elevated when compared to cases with solitary tumor ( $\sim 60$-fold higher than solitary hepatocellular carcinoma). As many studies have shown that intrahepatic satellite nodules in hepatocellular carcinoma are mostly metastases of the primary lesion, ${ }^{14,15}$ our finding may be interpreted as indicating a biological role for $Z Y X$ in hepatocellular carcinoma tumor cell disseminations. We have also shown $Z Y X$ overexpressions to 

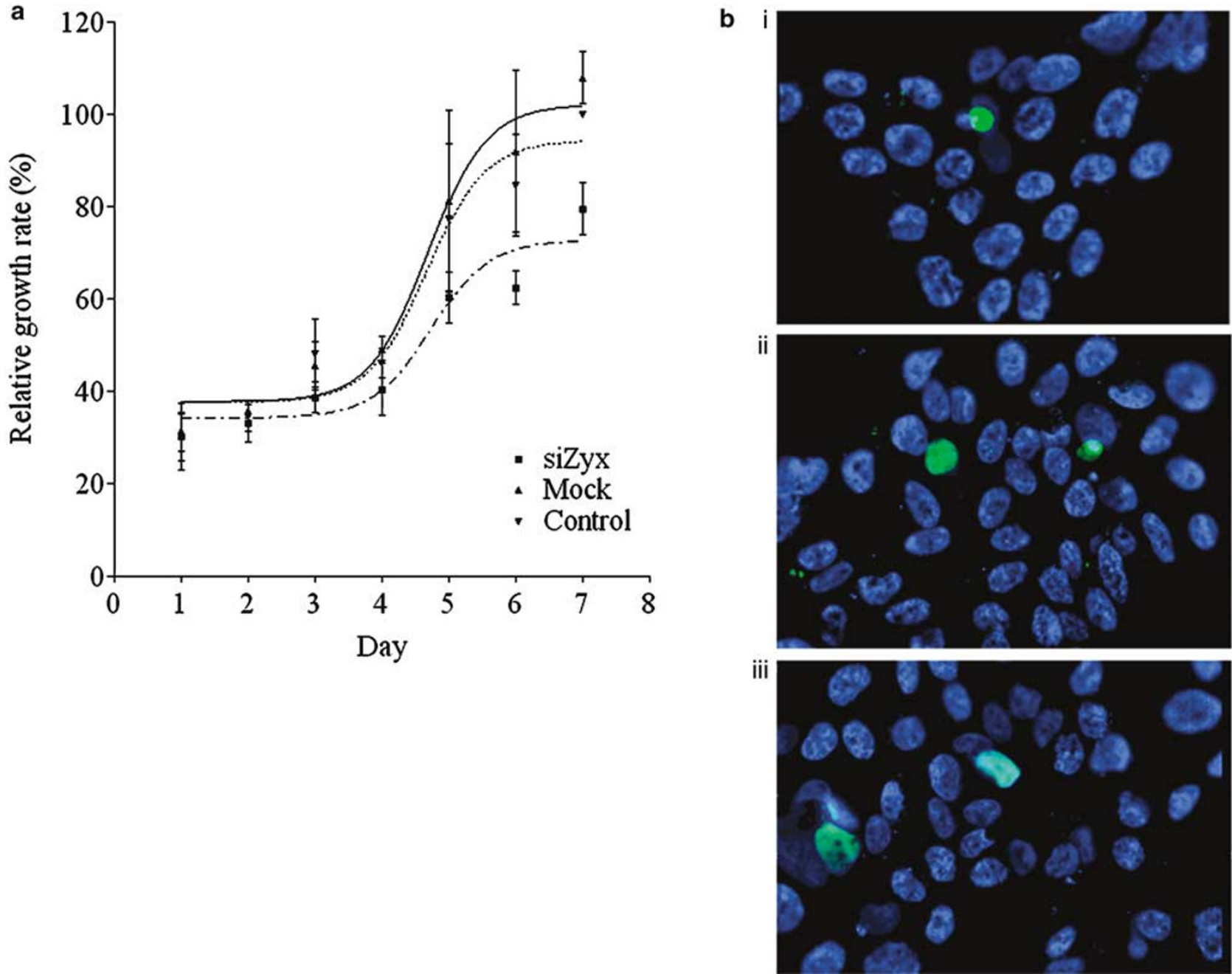

Figure 2 Effect on cell growth upon siZYX transfection. (a) A reduced growth rate by $\sim 30 \%$ was suggested in cells with suppressed $Z Y X$ expression. (b) Apoptotic status in cells transfected with (i) siZYX was found similar to that observed in (ii) mock and (iii) control experiments. Cells undergoing apoptosis were indicated by green fluorescent signals and nuclei were stained blue with $4^{\prime}, 6$-diamidino-2phenylindole.
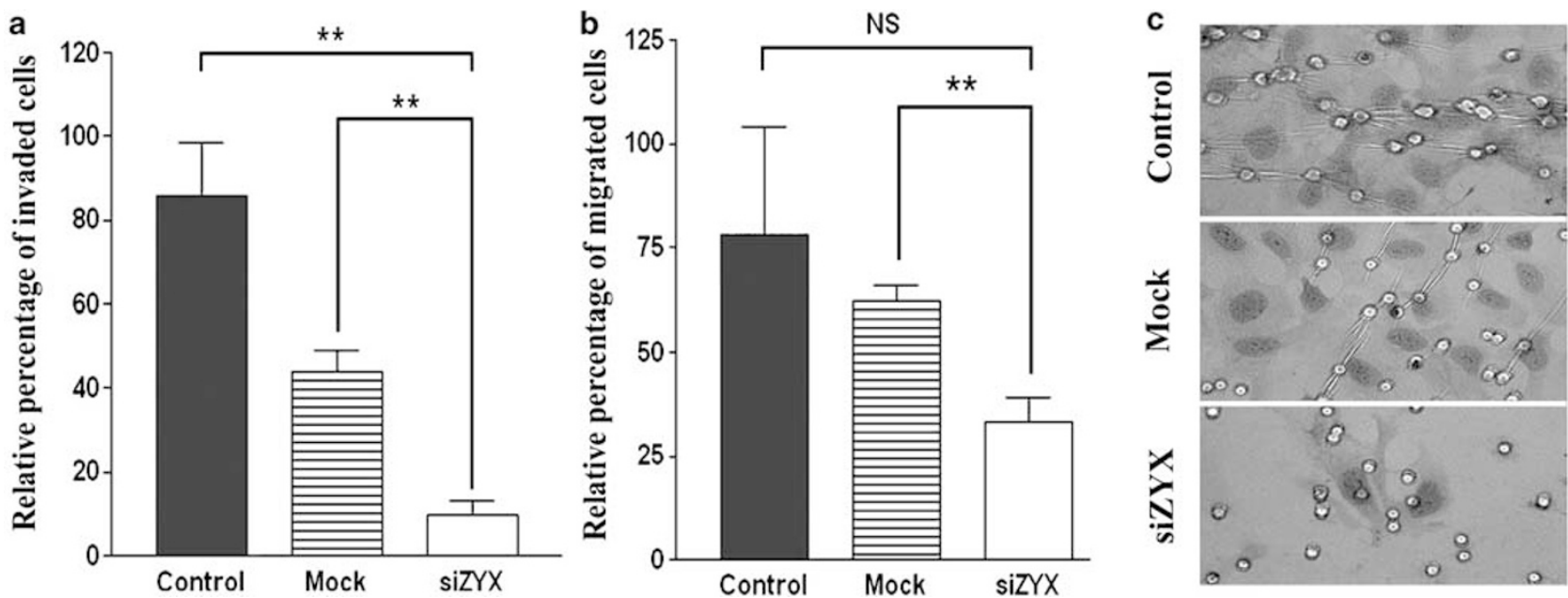

Figure 3 Effect on cell migration and cellular invasion upon $Z Y X$ expression knockdown. (a) A significant reduction in cell invasion through the extracellular matrix was observed in siZYX-transfected cells. ${ }^{* *} P<0.005$. (b) In cell migration assay, a significant reduction was also observed in cells with $Z Y X$ expression knockdown. Representative images of cells invaded through the matrix gel coating to the underside of migration chamber are shown in (c). NS: not significant, ${ }^{*} P<0.005$. 
be more common in those patients who developed recurrences following curative hepatectomy.

$Z Y X$ is a zinc-binding phosphoprotein $(\sim 61 \mathrm{kd})$ that is characterized by three LIM domains. It is abundant at focal adhesions and along the actin cytoskeleton. ${ }^{16}$ Our data suggest that $Z Y X$ suppression inhibits cell growth. Consistent with this observation $Z Y X$ expression has been shown to correlate directly with cell proliferation and in decreased the doubling time in melanoma cells. ${ }^{12}$ As there was no difference in the apoptotic status of $Z Y X$-deficient, mock and control cells, the effect of $Z Y X$ on hepatocellular carcinoma cell growth is unlikely to be mediated by antiapoptotic mechanisms. The ability of $Z Y X$ to enhance tumor growth may be attributable to the LIM domains, which have previously been shown to regulate cell proliferation and differentiation. ${ }^{17} \mathrm{We}$ also examined the role of $Z Y X$ in hepatocellular carcinoma cell invasion and migration, since $Z Y X$ has a reported role in mediating cell motility in vitro. ${ }^{18}$ Following $Z Y X$ expression knockdown, the ability of hepatocellular carcinoma cells to pass through the extracellular matrix and to migrate towards the chemo-attractant fetal bovine serum were both attenuated. These results suggest that $Z Y X$ expression plays a role in hepatocellular carcinoma cell motility and potentially underlined its function in tumor cell dissemination.

There is evidence to suggest that ZYX is recruited from cytoplasm to integrin-mediated focal complexes where it transforms the focal complexes into a focal adhesion. ${ }^{19}$ It is therefore possible that ZYX affects hepatocellular carcinoma cell migration through its interaction with integrins at sites of focal adhesions. Furthermore, it has been reported that ZYX is rapidly mobilized from the focal adhesions to actin stress fibers where it can mediate cell migration through actin tractions. ${ }^{18,20}$ Taken together, our data suggest that ZYX may have enhanced hepatocellular carcinoma cell migration and intravasation through its action on actin cytoskeleton and promoted cell dissemination as a part of the integrin-signaling pathways.

In the current study, our integrated mapping analyses have demonstrated the efficacy of targeting candidate genes within aberrant genomic loci. The characterization of $Z Y X$-deficient hepatocellular carcinoma cell has led to the recognition of its potential role in cell motility, invasion and proliferation. Further investigations into the molecular pathways of ZYX and its interaction with actin cytoskeleton should shed light on the metastatic behavior of hepatocellular carcinoma.

\section{Acknowledgements}

This work was supported by an Earmarked Grant from the Hong Kong Research Grants Council (Ref. No.: CUHK 4467/03M) and a Direct Grant from the
Chinese University of Hong Kong, and in part by a Central Allocation Grant from the Hong Kong Research Grants Council (Ref. No.: CUHK 2/02C).

\section{References}

1 Marchio A, Meddeb M, Pineau P, et al. Recurrent chromosomal abnormalities in hepatocellular carcinoma detected by comparative genomic hybridization. Genes Chromosomes Cancer 1997;18:59-65.

2 Wong N, Lai P, Lee SW, et al. Assessment of genetic changes in hepatocellular carcinoma by comparative genomic hybridization analysis: relationship to disease stage, tumor size, and cirrhosis. Am J Pathol 1999;154: $37-43$.

3 Kusano N, Shiraishi K, Kubo K, et al. Genetic aberrations detected by comparative genomic hybridization in hepatocellular carcinomas: their relationship to clinicopathological features. Hepatology 1999;29: 1858-1862.

4 Sy SM, Wong N, Lai PB, et al. Regional overrepresentations on chromosomes $1 \mathrm{q}$, $3 \mathrm{q}$ and $7 \mathrm{q}$ in the progression of hepatitis B virus-related hepatocellular carcinoma. Mod Pathol 2005;18:686-692.

5 Shiraishi K, Okita K, Harada T, et al. Comparative genomic hybridization analysis of genetic aberrations associated with development and progression of biliary tract carcinomas. Cancer 2001;91:570-577.

6 Pei J, Balsara BR, Li W, et al. Genomic imbalances in human lung adenocarcinomas and squamous cell carcinomas. Genes Chromosomes Cancer 2001;31:282-287.

7 Strohmeyer DM, Berger AP, Moore II DH, et al. Genetic aberrations in prostate carcinoma detected by comparative genomic hybridization and microsatellite analysis: association with progression and angiogenesis. Prostate 2004;59:43-58.

8 Weber RG, Sabel M, Reifenberger J, et al. Characterization of genomic alterations associated with glioma progression by comparative genomic hybridization. Oncogene 1996;13:983-994.

9 Beheshti B, Braude I, Marrano P, et al. Chromosomal localization of DNA amplifications in neuroblastoma tumors using cDNA microarray comparative genomic hybridization. Neoplasia 2003;5:53-62.

10 Chan KY, Wong N, Lai PB, et al. Transcriptional profiling on chromosome 19p indicated frequent downregulation of ACP5 expression in hepatocellular carcinoma. Int J Cancer 2005;114:902-908.

11 Chen MF, Hwang TL, Jeng LB, et al. Postoperative recurrence of hepatocellular carcinoma. Two hundred five consecutive patients who underwent hepatic resection in 15 years. Arch Surg 1994;129:738-742.

12 van der Gaag EJ, Leccia MT, Dekker SK, et al. Role of zyxin in differential cell spreading and proliferation of melanoma cells and melanocytes. J Invest Dermatol 2002;118:246-254.

13 Sanchez-Carbayo M, Socci ND, Charytonowicz E, et al. Molecular profiling of bladder cancer using cDNA microarrays: defining histogenesis and biological phenotypes. Cancer Res 2002;62:6973-6980.

14 Morimoto O, Nagano H, Sakon M, et al. Diagnosis of intrahepatic metastasis and multicentric carcinogenesis by microsatellite loss of heterozygosity in patients with multiple and recurrent hepatocellular carcinomas. J Hepatol 2003;39:215-221. 
15 Ng IO, Guan XY, Poon RT, et al. Determination of the molecular relationship between multiple tumour nodules in hepatocellular carcinoma differentiates multicentric origin from intrahepatic metastasis. J Pathol 2003;199:345-353.

16 Fradelizi J, Noireaux V, Plastino J, et al. ActA and human zyxin harbour Arp2/3-independent actin-polymerization activity. Nat Cell Biol 2001;3:699-707.

17 Gill GN. The enigma of LIM domains. Structure 1995; 3:1285-1289.
18 Drees BE, Andrews KM, Beckerle MC. Molecular dissection of zyxin function reveals its involvement in cell motility. J Cell Biol 1999;147:1549-1560.

19 Zaidel-Bar R, Cohen M, Addadi L, et al. Hierarchical assembly of cell-matrix adhesion complexes. Biochem Soc Trans 2004;32:416-420.

20 Yoshigi M, Hoffman LM, Jensen CC, et al. Mechanical force mobilizes zyxin from focal adhesions to actin filaments and regulates cytoskeletal reinforcement. J Cell Biol 2005;171:209-215. 